\title{
The Correspondence between the Molecular Orbital and Differential Ionization Energies Methods*
}

\author{
I. The Hydrogen Halides \\ RiCARDo Ferreira and JOHN K. BATES $\star \star$ \\ Chemistry Department, Earlham College, Richmond, Indiana 47374 \\ Received December 16, 1968/September 23, 1969
}

\begin{abstract}
The correspondence between Self-Consistent Hückel MO methods and Differential Ionization Energies methods is discussed in terms of the approximations used for the diagonal matrix elements. The two methods are shown to be equivalent if electronic correlation is neglected. Ground-state properties of the hydrogen halides are calculated by these simple methods and shown to be in good overall agreement with experimental data.

Die Übereinstimmung zwischen selbstkonsistenten Hückel MO-Methoden und Methoden der Differentiellen Ionisierungsenergien wird in Termen solcher Näherungen diskutiert, die für die diagonalen Matrixelemente benutzt werden. Es wird gezeigt, daß die beiden Methoden äquivalent sind, wenn die Elektronenkorrelation vernachlässigt wird. Grundzustandseigenschaften der "hydrogen halides" werden mit diesen einfachen Methoden ausgerechnet und zeigen sich in überall guter Übereinstimmung mit experimentellen Daten.

La correspondance entre les méthodes SCF Hückel et d'énergie d'ionisation différentielle est discutée en fonction des approximations utilisées pour les éléments de matrice diagonaux. Les deux méthodes sont équivalentes si la corrélation électronique est négligée. Les propriétés de l'état fondamental des acides halogénés sont calculées par ces méthodes simples et l'on constate un accord raisonnable avec les données expérimentales.
\end{abstract}

The purpose of this paper is to analyze the correspondence between SelfConsistent Hückel (SCHMO) methods [1-3] and the Differential Ionization Energies (DIE) calculations [4-7]. This problem has been dealt with by Jørgensen, Horner, Hatfield, and Tyree [4], and by Klopman [8], but it is presented here in a more general way, together with calculations for the ground-state properties of the hydrogen halides. These can be compared with SCF-LCAO-MO calculations of $\sigma$-bonded systems [9-11].

\section{Self-Consistent Hückel Methods}

Let $\varphi_{i}$ and $\varphi_{j}$ be the valence orbitals of atoms A and B, respectively. We describe a doubly occupied $\mathrm{MO}$ as:

$$
\Psi_{m}=c_{i} \varphi_{i}+c_{j} \varphi_{j}
$$

* Work supported by the Research Corporation and the National Science Foundation (Grant GY 2657/67).

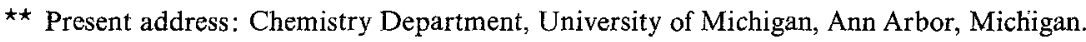


The operator of $H_{i i}=\left\langle\varphi_{i} \mathscr{H} \varphi_{i}\right\rangle$ includes the interaction of one electron centered in $\varphi_{i}$ with all other nuclei and electrons, including the other electron in the molecular orbital $\Psi_{m}$. The diagonal elements may be partitioned by Mulliken's approximation [12]:

$$
H_{i i}=\varepsilon_{i}+V_{i j}+c_{i}^{2} J_{i i}+c_{j}^{2} J_{i j}
$$

where $\varepsilon_{i}=\left\langle\varphi_{i}^{*}(1)\left|-\frac{1}{2} \nabla_{1}^{2}-\frac{1}{r_{1 i}}\right| \varphi_{i}(1)\right\rangle$ is the atomic one-electron eigenvalue. $\varepsilon_{i}$ is equal to $-I_{i}$, that is, to the VOIP (or VSIP) corresponding to the atomic orbital $\varphi_{i} . V_{i j}$ lumps together the core integrals $\left\langle\bar{u}_{j} \varphi_{i}^{*} \varphi_{i}\right\rangle$ and $J_{i i}$ and $J_{i j}$ are the two-electron Coulomb integrals. We further assume $[13,14]$ that $J_{i i}=I_{i}-A_{i}$, where $A_{i}$ is the valence-state electron affinity (VSEA). Eq. (2) can be written:

$$
H_{i i}=-I_{i}+c_{i}^{2}\left(I_{i}-A_{i}\right)+V_{i j}+c_{j}^{2} J_{i j} .
$$

The first two terms in the right-hand side of (3) are the atomic terms, the others the molecular terms of $H_{i i}$. The latter corresponds to an interatomic Coulomb correction, and has been called the Madelung-Jørgensen potential [15]. The charge dependence of the parameters $H_{i i}$ and $H_{i j}$ must be included in the differentiations leading to the minimum-energy condition. This important point has been recently stressed by Harris [16].

The form of the atomic terms in (3) is identical to Moffitt's approximation [13], and it is also consistent with the SCF matrix element formulation [1]. The atomic orbital energy function derived from the atomic terms of Eq. (3) is:

$$
\varepsilon_{i}\left(n_{i}\right)=-I_{i} n_{i}+1 / 4 n_{i}^{2}\left(I_{i}-A_{i}\right)
$$

where $n_{i}=2 c_{i}^{2}$ is the occupation number of orbital $\varphi_{i}$. The quantum mechanical expression for the energy of an isolated atomic orbital is, on the other hand:

$$
\varepsilon_{i}\left(n_{i}\right)=-I_{i} n_{i}+1 / 2 n_{i}\left(n_{i}-1\right)\left(I_{i}-A_{i}\right) .
$$

The difference (4)-(5) corresponds to the right-left correlation energy [17], which is entirely neglected in simple MO theory. It will be shown in Sect. 3 that Eq. (4) is the only atomic energy function that leads to a complete equivalence between the SCHMO and the DIE methods.

We assume that the point charge description [18] holds and if $c_{i}^{2}=1-c_{j}^{2}$, Eq. (3) transforms to:

$$
H_{i i}=-I_{i}+c_{i}^{2}\left(I_{i}-A_{i}\right)-c_{i}^{2} \frac{e^{2}}{r_{i j}}
$$

The off-diagonal matrix elements are calculated by an equation of the type:

$$
H_{i j}=K S_{i j}\left(H_{i i}+H_{j j}\right) / 2 \text {. }
$$

The procedure, however, is not strictly a Wolfsberg-Helmholz calculation because overlap integrals are neglected in the secular equations. As a result, the value of 
$K$ in Eq. (7) can be freely varied without the associated dangers of inversion of the energy levels [19-23].

Eq. (6) is the correct diagonal matrix element for the calculation of one-electron properties of $\mathrm{AB}$ systems, such as ionization potentials. Bond energies, force constants, dipole moments, etc., are, however, electron-pair properties and expression (6) must be modified. From the cycle:

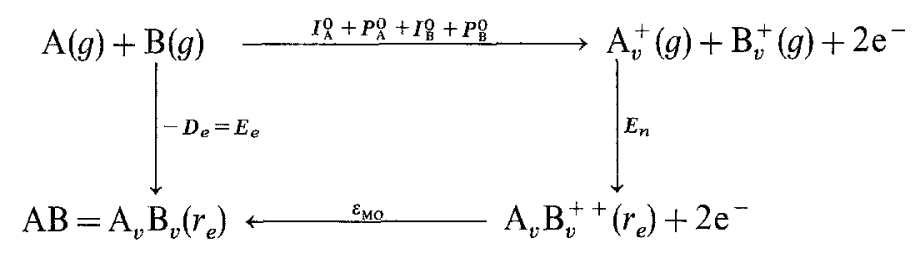

we have:

$$
E_{e}=-D_{e}=\varepsilon_{\mathrm{MO}}+E_{n}+I_{\mathrm{A}}^{0}+P_{\mathrm{A}}^{0}+I_{\mathrm{B}}^{0}+P_{\mathrm{B}}^{0} .
$$

With the usual conventions, $D_{e}>0 ; E_{e}$ is simply $-D_{e}$, and binding occurs only if $\varepsilon_{\mathrm{MO}}<0$ and $\left|\varepsilon_{\mathrm{MO}}\right|>\left(E_{n}+I_{\mathrm{A}}^{0}+P_{\mathrm{A}}^{0}+I_{\mathrm{B}}^{0}+P_{\mathrm{A}}^{0}\right) . E_{n}$ is the core-core repulsion energy; in our calculations $E_{n}=\frac{Z_{\mathrm{A}} Z_{\mathrm{B}}}{r_{\mathrm{AB}}}$, where $Z_{\mathrm{A}}$ and $Z_{\mathrm{B}}$ are the effective charges seen by a unit charge at the distance $r_{\mathrm{AB}}$ from $\mathrm{A}$ and $\mathrm{B}$, respectively. We may write

$$
E_{n}=\frac{Z_{\mathrm{A}} Z_{\mathrm{B}}}{r_{\mathrm{AB}}}=\frac{e^{2}}{r_{\mathrm{AB}}}+\frac{\left(Z_{\mathrm{A}} Z_{\mathrm{B}}-e^{2}\right)}{r_{\mathrm{AB}}}
$$

where the first term in the right-hand side is the Coulomb repulsion between $\mathrm{A}_{v}^{+}$ and $\mathrm{B}_{v}^{+}$, and $\frac{\left(Z_{\mathrm{A}} Z_{\mathrm{B}}-e^{2}\right)}{r_{\mathrm{AB}}}$ is a penetration term. $\mathrm{A}_{v}$ and $\mathrm{B}_{v}$ refer to the valence states, and $P_{\mathrm{A}}^{0}$ and $P_{\mathrm{B}}^{0}$ are the promotion energies. Since the $H_{i i}$ and $H_{i j}$ integrals are one-electron integrals, the Hamiltonian for the process $\mathrm{AB} \rightarrow \mathrm{AB}^{+}+\mathrm{e}^{-}$is different from the Hamiltonian for the process $\mathrm{AB}^{+} \rightarrow \mathrm{AB}^{++}+\mathrm{e}^{-}$. In the latter, there is no screening potential from the second electron [12]. We have:

$$
\begin{aligned}
& H_{i i}(1)=-I_{i}+c_{i}^{2}\left(I_{i}-A_{i}\right)-c_{i}^{2} \frac{e^{2}}{r_{i j}}, \\
& H_{i i}(2)=-I_{i}-\frac{e^{2}}{r_{i j}} .
\end{aligned}
$$

The diagonal matrix elements to be used for the calculation of two-electron properties is the average value:

$$
H_{i i}=-I_{i}+\frac{c_{i}^{2}}{2}\left(I_{i}-A_{i}\right)-\frac{\left(1+c_{i}^{2}\right)}{2} \frac{e^{2}}{r_{i j}} .
$$

Eq. (10) and (7) give the correct one-electron integrals, and for the doubly occupied MO:

$$
\varepsilon_{\mathrm{MO}}=2\left[c_{i}^{2} H_{i i}+c_{j}^{2} H_{j j}+2 c_{i} c_{j} H_{i j}\right] .
$$


For the usual equilibrium internuclear distance, the terms $\left(I_{i}-A_{i}\right)$ and $\frac{e^{2}}{r_{i j}}$ are of comparable magnitude and this [8] explains the success of SCCC-LCAOMO methods in which one assumes [4] that $H_{i i}=-(\text { VSIP) })_{i}$. Also, because Eq. (10) shows a small dependence of $H_{i i}$ on $c_{i}^{2}$, we can explain the use of the hydride or neutral atom ionization energies for the diagonal elements of ligand ions in complexes [24].

\section{The Differential Ionization Energies Method}

In this method [4-7], the bond energy is given by a sum of atomic and interatomic terms expressed as functions of the atomic charges and, for the latter terms, of the bond distances. The relation between the occupation numbers of the orbitals $\varphi_{i}$ and $\varphi_{j}$ and the bond ionicity $\chi$ depends on the way the orbital populations are defined. In our case, $S_{i j}=0$, and since $n_{i}+n_{j}=2$, we have $\chi=n_{i}-1=c_{i}^{2}-c_{j}^{2}$ $=2 c_{i}^{2}-1$. For the atomic energy terms, it is customary $[4,5,7,8,25,26]$ to use Eq. (5). We will show in the next section that the DIE method is equivalent to the SCH-MO method only if we use Eq. (4) for the atomic orbital energy terms. This means that for the separated atoms, or for a purely covalent bond $(x=0)$, we are including too much electron repulsion. We also must change coordinates since now $\varepsilon_{i}(0)=+I_{i}, \varepsilon_{i}(1)=1 / 4\left(I_{i}-A_{i}\right)$, and $\varepsilon_{i}(2)=-A_{i}$, corresponding to $\chi=-1,0$, and +1 . Since $n_{i}=2 c_{i}^{2}=x+1$ and $n_{j}=2 c_{j}^{2}=1-x$, Eq. (4) can now be written:

$$
\begin{aligned}
& \varepsilon_{i}(x)=1 / 4\left(I_{i}-A_{i}\right)-1 / 2\left(I_{i}+A_{i}\right) x+1 / 4\left(I_{i}-A_{i}\right) x^{2}=\varepsilon_{i}\left(n_{i}\right)+I_{i}, \\
& \varepsilon_{j}(x)=1 / 4\left(I_{i}-A_{i}\right)+1 / 2\left(I_{i}+A_{j}\right) x+1 / 4\left(I_{j}-A_{j}\right) x^{2}=\varepsilon_{j}\left(n_{j}\right)+I_{j} .
\end{aligned}
$$

In this paper, we will suppose that $\left|F_{i i}\right|>\left|F_{j j}\right|\left(F_{i i}\right.$ represents the Hartree-Fock matrix elements; see Sect. 3), and hence $x$ is always positive. The interatomic term is described by the sum of a covalent and an ionic bond energy, each one multiplied by the corresponding bond-order $[7,27]$. The covalent bond order is $2 c_{i} c_{j}=\left(1-x^{2}\right)^{1 / 2}$, the ionic bond order is $x^{2}$. The ionic bond energy is $-\frac{e^{2}}{r_{i j}}$. Several approximations can be used for the covalent bond energy $E_{c}$, and our particular choice will be discussed in Sect. III. Thus, the bond energy function is:

$$
E_{e}(x)=\left(1-x^{2}\right)^{1 / 2} E_{c}-x^{2} \frac{e^{2}}{r_{i j}}+\varepsilon_{i}(x)+\varepsilon_{j}(x)+\mathscr{R} .
$$

$\varepsilon_{i}(x)$ and $\varepsilon_{j}(x)$ are given by Eq. (12a) and (12b), and $\mathscr{R}$ is the repulsive penetration term for the approaching atoms and given by $\mathscr{R}=\frac{Z_{a} Z_{b}-e^{2}}{r_{i j}}$.

For any reasonable value of $r_{i j}, E_{e}(x)$ is negative and, in fact, the bond energy is $D_{e}=-E_{e}\left(x_{e}\right)$.

Bond energies are calculated by minimizing $E(x)$ with respect to $x$. One must decide what approximation to use for $E_{c}$, the covalent bond energy. The arith- 
metic and geometrical mean rules cannot be used, because they include the penetration term, whereas in Eq. (13), this term is shown explicitely. We will show in the next section that the point charge approximation leads to $E_{c}=\left(2 H_{i j}-\frac{e^{2}}{r_{i j}} c_{i} c_{j}\right)$, where $H_{i j}$ is the off-diagonal matrix element. With this approximation, $E(x)$ may be written as:

$E(x)=\left[2 H_{i j}-\frac{e^{2}}{2 r_{i j}}\left(1-x^{2}\right)^{1 / 2}\right]\left(1-x^{2}\right)^{1 / 2}-x^{2} \frac{e^{2}}{r_{i j}}+\varepsilon_{i}(x)+\varepsilon_{j}(x)+\mathscr{R}$.

Accordingly:

$$
\begin{aligned}
\frac{d E(x)}{d x}= & -\frac{x}{\left(1-x^{2}\right)^{1 / 2}}\left(2 H_{i j}-x \frac{e^{2}}{r_{i j}}\right)-1 / 2\left(I_{i}+A_{i}\right)+1 / 2\left(I_{i}-A_{i}\right) x \\
& +1 / 2\left(I_{j}+A_{j}\right)+1 / 2\left(I_{j}-A_{j}\right) x=0
\end{aligned}
$$

and hence*

$$
x_{e}=\frac{X_{i}-X_{j}}{-2 H_{i j}\left(1-x^{2}\right)^{-1 / 2}-\frac{e^{2}}{r_{i j}}+1 / 2\left(I_{i}-A_{i}\right)+1 / 2\left(I_{j}-A_{j}\right)}
$$

where $X_{i}=1 / 2\left(I_{i}+A_{i}\right)$ and $X_{j}=1 / 2\left(I_{j}+A_{j}\right) . x_{e}$ is, of course, a function of $r_{i j}$. Eq. (15) is identical to Eq. (32) of Jenkins and Pedley [11], which shows the correspondence between the two methods. It should be pointed out that Eq. (15) was derived from Eq. (4) and corresponds to complete neglect of correlation. As a result, Eq. (15) gives too high values for $x_{e}$. Eq. (5) on the other hand overestimates correlation and leads to an equation identical to (15) except that the terms $(I-A)$ in the denominator appear with coefficients of one, instead of $1 / 2$.

The relative merits of the two approaches have been discussed by Baird, Sichel, and Whitehead [28].

\section{The Equivalence between the SCHMO and DIE Methods}

The equivalence between the self-consistent method in Hückel theory and the DIE method can be established from the fact that the following identity holds:

$$
\begin{aligned}
-D_{e}= & E(x)=\left(2 H_{i j}-1 / 2\left(1-x^{2}\right)^{1 / 2} \frac{e^{2}}{r_{i j}}\right)\left(1-x^{2}\right)^{1 / 2}-x^{2} \frac{e^{2}}{r_{i j}} \\
& +1 / 4(I-A)_{i}-1 / 2(I+A)_{i} x+1 / 4(I-A)_{i} x^{2}+1 / 4(I-A)_{j} \\
& +1 / 2(I+A)_{j} x+1 / 4\left(I_{j}-A_{j}\right) x^{2}+\frac{Z_{a} Z_{b}-e^{2}}{r_{i j}} \\
& \equiv 2 c_{i}^{2}\left[-I_{i}+c_{i}^{2} / 2(I-A)_{i}-\left(1+c_{1}^{2}\right) / 2 e^{2} / r_{i j}\right] \\
& +2 c_{j}^{2}\left[-I_{j}+c_{j}^{2} / 2(I-A)_{j}-\left(1+c_{j}^{2}\right) / 2 e / r_{i j}\right] \\
& +2\left(2 c_{i} c_{j}\right) H_{i j}+\frac{Z_{a} Z_{b}}{r_{i j}}+I_{\mathrm{A}}^{0}+P_{\mathrm{A}}^{0}+I_{\mathrm{B}}^{0}+P_{\mathrm{A}}^{0} .
\end{aligned}
$$


The identity (16) holds provided $x=c_{i}^{2}-c_{j}^{2}\left(S_{i j}=0\right)$. It also depends on the identification of $E_{c}$ with $\left[2 H_{i j}-c_{1} c_{2} \frac{e^{2}}{r_{i j}}\right]$. It is sometimes assumed that $E_{c}=2 H_{i j}$; but this is only true if we drop the Coulomb interatomic terms in the diagonal elements.

Harris [16] has recently drawn the attention to an important point: in minimizing $\varepsilon_{\mathrm{MO}}$ with respect to the variational coefficients the charge dependence of $H_{i i}$ and $H_{i j}$ must be included. That is, the correct eigenvalue equations are:

$$
c_{1}\left(F_{11}-\varepsilon\right)+c_{2}\left(F_{12}\right)=0, \quad c_{1}\left(F_{12}\right)+c_{2}\left(F_{22}-\varepsilon\right)=0
$$

where

$$
F_{11}=H_{11}+\frac{c_{1}^{2}}{2}\left[(1-A)_{1}-\frac{e^{2}}{r_{i j}}\right]=-I_{1}+c_{1}^{2}(I-A)-\frac{e^{2}}{r_{i j}} \frac{\left(1+2 c_{1}^{2}\right)}{2}
$$

and

$$
F_{12}=H_{12}+c_{1}^{2} \frac{K S_{12}}{4}\left[(I-A)_{1}-\frac{e^{2}}{r_{i j}}\right]+\frac{c_{2}^{2}}{4} K S_{12}\left[(I-A)-\frac{e^{2}}{r_{i j}}\right]
$$

which, on the one hand, corresponds exactly to the system of Eq. (9) of Harris [16] and, on the other hand, to the minimization procedures of the DIE method leading to Eq. (15) and hence to the energy function (13) above.

\section{The Hydrogen Halides: Results and Discussion}

The main points in the calculation of ground state properties of the hydrogen halides are as follows: $F_{11}, F_{22}$ and $F_{12}$ were given by $(17 \mathrm{a})$ and $(17 \mathrm{~b}) ; H_{12}$ is given by (7); the best value of $K$ in Eq. (7) was found by trial to be 0.4 . Since the calculation is not a Wolfsberg-Helmholz treatment, no special significance should be assigned to the chosen value of $K$. Overlap integrals in Eq. (7) are from Mulliken, Rieke, Orloff, and Orloff [29], and $S(4 p, 1 s)$ values are interpolated from $S(3 p, 1 s)$ and $S(5 p, 1 s)$. Self-consistent solutions of Roothaan Eq. (17) were attained by the usual procedures $[9,30]$. Core-core repulsions, $E_{n}$, are calculated by the method suggested by Pohl and Raff [10], $E_{n}=\frac{Z_{\mathrm{A}} Z_{\mathrm{B}}}{r_{i j}}$, where $Z_{\mathrm{A}}$ and $Z_{\mathrm{B}}$ are estimated from the Herman-Skillman atomic structure tables [31]. Valence state ionization energies and electron-affinities are from Hinze and Jaffe [32]. Calculations were made with an IBM 1130 computer.

Calculated and experimental [33-36] ground-state properties of the hydrogen halides are given in the table and the calculated potential curves for these four molecules are shown in Fig. 1. In general, the calculated values are in reasonable agreement with the experimental ones. The trend in dissociation energies, equilibrium internuclear distances, bond dipole moments, dipole moment functions and force constants is the correct one throughout the series HX. As predicted, 
Table

\begin{tabular}{llllll}
\hline & HF & HCl & HBr & HI & Ref. \\
\hline$D_{e}(\mathrm{calc})(\mathrm{eV})$ & 7.59 & 4.84 & 3.73 & 1.85 & \\
$D_{e}(\exp )(\mathrm{eV})$ & 6.08 & 4.47 & 3.80 & 3.10 & {$[33]$} \\
$R_{e}(\mathrm{calc})(\AA)$ & 0.87 & 1.30 & 1.48 & 1.64 & \\
$R_{e}(\exp )(\AA)$ & 0.917 & 1.275 & 1.414 & 1.604 & {$[33]$} \\
$\chi_{e}(\mathrm{calc})$ & 0.68 & 0.26 & 0.16 & 0.075 & \\
$\mu_{\text {prim }}(\mathrm{calc})(\mathrm{D})$ & 3.26 & 1.24 & 0.77 & 0.36 & \\
$\mu(\exp )(\mathrm{D})$ & 1.82 & 1.08 & 0.82 & 0.44 & {$[34]$} \\
$\left(\frac{\partial \mu}{\partial r}\right)_{r e}(\mathrm{calc})(\mathrm{D} / \AA)$ & +2.12 & +1.08 & +0.71 & +0.36 & \\
$\left(\frac{\partial \mu}{\partial r}\right)_{r e}(\exp )(\mathrm{D} / \AA)$ & +1.8 & \pm 1.0 & \pm 0.9 & \pm 0.2 & {$[35,36]$} \\
$k($ calc) $($ dynes $/ \mathrm{cm})$ & $20.2 \times 10^{5}$ & $4.6 \times 10^{5}$ & $2.5 \times 10^{5}$ & $1.1 \times 10^{5}$ & \\
$k($ exp) $($ dynes $/ \mathrm{cm})$ & $9.6 \times 10^{5}$ & $5.1 \times 10^{5}$ & $4.1 \times 10^{5}$ & $3.1 \times 10^{5}$ & {$[33]$} \\
\hline
\end{tabular}

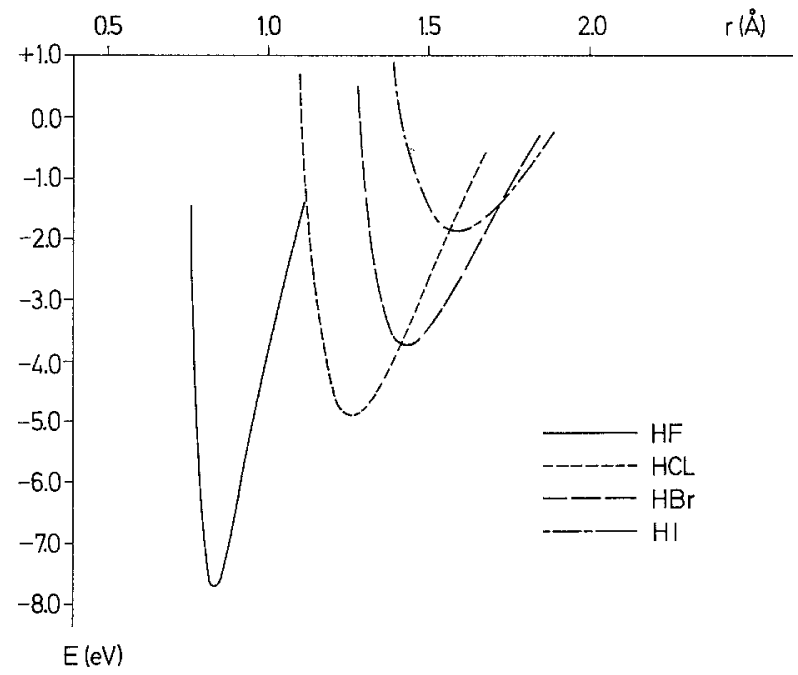

Fig. 1. Potential curves of the hydrogen halides

function (4) tends to introduce a high ionicity, and this is reflected in the high values of the calculated dipole moments of $\mathrm{HF}$ and $\mathrm{HCl}$. Actually, these values represent the primary moments only, the total dipole being the sum of the primary and the homopolar moments. The calculated values of $\left(\frac{\partial \mu}{\partial r}\right)_{r_{e}}$ are in good agreement with the experimental ones. They are also in general accord with 
dipole moment functions calculated (for $\mathrm{HF}$ and $\mathrm{HCl}$ ) using advanced $\mathrm{SCF}$ techniques [37]. There is little doubt that the sign of $\left(\frac{\partial \mu}{\partial r}\right)$ at the equilibrium distance is positive and that for ordinary chemical bonds the stationary value of the bond moment occurs at distances larger than the equilibrium internuclear distances. This is true in our calculation although $\left(\frac{\mathrm{d} x}{\mathrm{~d} r}\right)$ is always negative.

The calculated bond energies and force constants are too large for HF, and too small for HI. There is little doubt that the point charge approximation as used here over-emphasizes the Madelung-Jørgensen energy terms in $F_{11}$ [8]. Because of polarization effects, the charges should not be located at the nuclear centers. Corrections along these lines would undoubtedly produce better results, notably for hydrogen fluoride, but at the expense of one more arbitrary parameter.

Acknowledgement. We wish to thank Professor G. Klopman for helpful discussions and Dr. H. D. B. Jenkins, Dr. M. Giambiagi, and Professor C. K. Jørgensen for the benefit of correspondence.

\section{References}

1. Cusachs, L. C., Reynolds, J. W.: J. chem. Physics 43, 60 (1965).

2. Carroll, D. G., Armstrong, A. T., McGlynn, S. P.: J. chem. Physics 44, 1865 (1966).

3. Rein, R., Fukuda, N., Win, H., Clarke, G. A., Harris, F. E.: J. chem. Physics 45, 4743 (1966).

4. Jørgensen, C. K., Horner, S. M., Hatfield, W. E., Tyree, S. Y., Jr.: Int. J. quant. Chemistry 1, 191 (1967).

5. - Orbitals in Atoms and Molecules, Chapter 1 and 7, New York: Academic Press 1962.

6. Pearson, R. G., Gray, H. B.: Inorg. Chem: 2, 358 (1963).

7. Ferreira, R.: J. physic Chem. 68, 2240 (1964).

8. Klopman, G.: J. Amer. chem. Soc. 86, 1463, 4550 (1964); 87, 3300 (1965).

9. Pohl, H. A., Rein, R., Appel, K.: J. chem. Physics 41, 3385 (1964).

10. 一, Raff, L. M.: Int. J. quant. Chemistry 1, 577 (1967).

11. Jenkins, H. D. B., Pedley, J. B.: Theoret. chim. Acta (Berl.) 3, 309 (1965).

12. Mulliken, R. S.: J.Chim. physique 46, 497, 675 (1949).

13. Moffitt, W.: Proc. Roy. Soc. (London) A 196, 510 (1949).

14. Pariser, R.: J. chem. Physics 21, 568 (1953).

15. Ferreira, R.: Advances chem. Physics 13, 55 (1967).

16. Harris, F. E.: J. chem. Physics 48, 4027 (1968).

17. Slater, J. C.: Quantum theory of matter, 2 nd, ed, p. 448, New York: McGraw-Hill Book Co. 1968.

18. Pople, J. A.: Trans. Faraday Soc. 49, 1375 (1953).

19. Del Re, G.: Nuovo Cimento 17, 644 (1960).

20. Hoffman, R.: J. chem. Physics. 39, 1397 (1963); 40, 2745 (1964).

21. Gonzales, H. C., De Giambiagi, M. S., Giambiagi, M.: Theoret. chim. Acta (Berl.) 6, 257 (1966).

22. Ohno, K.: Advances quant. Chem. 3, 314 (1967).

23. Cusachs, L. C.: Int. J. quant. Chemistry Supplement 1, 419 (1967).

24. Viste, A., Gray, H. B.: Inorg. Chem. 3, 1113 (1964).

25. Pritchard, H. O., Sumner, F. H.: Proc. Roy. Soc. (London) A 235, 136 (1956).

26. Hinze, J., Whitehead, M. A., Jaffe, H. H.: J. Amer. chem. Soc. 85, 148 (1963).

27. Mulliken, R. S.: J. chem. Physics 23, 1841 (1955).

28. Baird, N. C., Sichel, J. M., Whitehead, M. A.: Theoret. chim. Acta (Berl.) 11, 38 (1968).

29. Mulliken, R. S., Rieke, C. A., Orloff, D., Orloff, H.: J. chem. Physics 17, 1248 (1949).

30. Carbo, R., De Carbo, C. A., Molina, J.: Afinidad 22, 403 (1965). 
31. Herman, F., Skillman, S.: "Atomic Structure Calculations", New Jersey: Prentice-Hall Inc. 1963.

32. Hinze, J., Jaffe, H. H.: J. Amer. chem. Soc. 84, 540 (1962); J. chem. Physics 38, 1834 (1963).

33. Herzberg, G.: "Spectra of Diatomic Molecules", Princeton: D. Van Nostrand 1961.

34. Nelson, R. D., Jr., Lide, D. R., Jr., Maryott, A. A.: "Selected Values of Electric Dipole Moments for Molecules in the Gas Phase", NBS Reference Data Series 10, Washington: D. C. 1967.

35. Bartholme, E.: Z. physik. Chem. B 23, 131 (1933).

36. Magnuson, D. W.: Oak Ridge National Lab., Report K-1180 (1954).

37. Nesbet, R. K.: J. chem. Physics 36, 1518 (1962); 41, 100 (1964).

Prof. Dr. R. Ferreira

Department of Chemistry

Earlham College

Richmond, Indiana 47374, U.S.A. 